\section{Zur Diagnose GÖRK reichen Symptome allein nicht aus}

\begin{abstract}
— Der gastroösophageale Reflux (GÖR) ist ein normaler, physiologischer Prozess bei Säuglingen. Die Abgrenzung zur gastroösophagealen Refluxkrankheit (GÖRK) ist aufgrund der unspezifischen Symptome nicht immer einfach. Nun sollen neue Leitlinien zur GÖRK die tägliche Arbeit der Pädiater erleichtern. Demnach liegt eine GÖRK vor, wenn der Reflux von Mageninhalt Beschwerden oder Komplikationen verursacht. „Wichtig ist, dass die Symptome das Kind, nicht die Betreuungsperson beeinträchtigen!“, betonte Prof. Dr. Sibylle Koletzko aus München.

Auch weil Kinder erst ab etwa acht Jahren in der Lage sind, ihre Beschwerden zuverlässig zu lokalisieren, kann die Diagnose GÖRK bei einem Säugling, einem jungen oder kognitiv beeinträchtigten älteren Kind nicht nur aufgrund der Anamnese gestellt werden. „GÖRK muss sich nicht unbedingt als Schmerz äußern, möglich sind z.B. auch chronischer Husten, Nahrungsverweigerung oder Gedeihstörungen“, sagte Koletzko.
\end{abstract}

Klinisch lässt sich die GÖRK von der Kuhmilchproteinallergie schwer unterscheiden, zudem treten die Krankheiten überzufällig häufig gemeinsam auf (40\%). „Bei Kuhmilchallergie kann der Magenausgang verschwollen sein, so dass auch Wasser und nicht Kuhmilchprotein-haltige Beikost erbrochen wird“, erklärte Koletzko. Klarheit schafft in der Regel eine Kuhmilchprotein-freie Eliminationsdiät. Bei ungestillten Säuglingen eignet sich hierfür eine extensiv hydrolysierte Eiweißformula oder eine Aminosäurenformula (z. B. Neocate ${ }^{\circledR}$ ). „Eine Therapie mit Protonenpumpenhemmern sollte streng indiziert sein, da sie das Risiko für eine Sensibilisierung gegen Nahrungsmittel und eine manifeste Nahrungsmittelallergie erhöht“, fügte Koletzko hinzu. mha

Roundtable-Gespräch „Gastroösophageale Refluxkrankheit im Kindesalter", München 14. Juli 2010. Veranstalter: Pfrimmer Nutricia, Erlangen

\title{
13-valenter Pneumokokken-Impfstoff schützt am besten
}

_Die akute Otitis media ist der häufigste
Anlass für einen Besuch in der kinderärzt-
lichen Praxis. Die schwersten Symptome
werden in der Regel durch Pneumokokken
verursacht, berichtete Prof. Dr. Dr. Dr. Dieter
Adam aus München. So beobachte man im-
mer wieder Komplikationen wie Trommel-
fellperforation, Paukenerguss, Mastoiditis
oder Hörminderung, schlimmstenfalls sogar
lebensbedrohliche Meningitiden oder Hirn-
abszesse. Eine noch laufende Multizenter-
studie, für die Säuglinge und Kleinkinder mit
spontan perforierter akuter Otitis media
rekrutiert wurden, untersucht derzeit erst-
mals das Erregerspektrum in Deutschland.
Eine auf dem diesjährigen ESPID-Kongress
vorgestellte Zwischenauswertung von 459
Patienten zeigt: Bei 191 identifizierten Kei-
men ging rund ein Viertel der bakteriellen
Otitis media auf das Konto von Strepto-
coccus pneumoniae, wobei die Serotypen
3 (36\%) und 19A (21\%) dominierten. „Eine
Impfung muss deshalb primär gegen Pneumokokkeninfektionen schützen", hob Adam hervor.

Wie Studienleiter Dr. Andreas Busse, Tegernsee, aufführte, enthält der seit Ende 2009 zugelassene neue 13-valente Impfstoff PCV13 (Prevenar $13^{\circledR}$ ) als einzige PneumokokkenKonjugatvakzine auch Antigene gegen die Serotypen 3 und 19A und zeigt deshalb mit $70 \%$ die höchste Abdeckungsrate. Im Vergleich dazu betrage die Coverage für den 7und 10-valenten Impfstoff nur noch 9 bzw. $13 \%$., Es ist zu erwarten, dass der 13 -valente Impfstoff einen wesentlichen Beitrag zur Prophylaxe der schweren akuten Otitis media leisten wird“, resümierte Busse. mju

Post-ESPID-Pressegespräch „Akute Otitis media: Stellenwert in der Praxis des Kinderarztes“. München, 26. Mai 2010. Veranstalter: Wyeth Pharma, ein Unternehmen der Pfizer-Gruppe,Münster

\section{Flauschiger Praxishelfer}

Gemeinsam mit der Firma Steiff hat das Familienunternehmen bene-Arzneimittel einen Helfer entwickelt, der kleinen Patienten die Angst vor gefürchteten Untersuchungen und medizinischen Behandlungen nimmt: den ben-u-ron-MediTeddy. Mehrere Bereiche des Fells lassen sich wegklappen und bieten so einen Einblick in das Innere des Körpers. Für Kinder verständlich kann der Arzt am Beispiel der Darstellung die menschlichen Organe und Körperbereiche erläutern. Außerdem kann er verschiedene Untersuchungen zunächst beim MediTeddy durchführen. Den ben-u-ron-MediTeddy können Praxen unter mediteddy@bene-arzneimittel.de für einen Praxistarif von 39 Euro bestellen.

Nach Informationen von bene-Arzneimittel, München

\section{Halsschmerztablette mit Historie}

Tonsipret ${ }^{\circledR}$ gibt es nun schon seit 15 Jahren. Die auf dem deutschen Markt einzige pflanzliche Halsschmerztablette mit Wirkstoffen aus Arzneipaprika, Kermesbeere und Pockholz wirkt analgetisch, antiphlogistisch, immunmodulierend und diaphoretisch. Das Kombinationspräparat ist als Tabletten oder Tropfen erhältlich. Tonsipret ${ }^{\circledR}$ ist sehr gut verträglich und daher bereits für Kinder $a b$ einem Jahr geeignet.

Nach Informationen von Bionorica, Neumarkt

\section{Risikolose Impfstofflagerung}

Der Impfstoffversender a1.direkt - bisher bekannt als A1-VersandApotheke - bietet ab sofort einen neuen Premium-Service für Pädiater an: Eine neue Impfstoffversicherung stellt sicher, dass von a1.direkt gelieferte und in der Praxis gelagerte Impfstoffe keinen größeren Temperaturschwankungen ausgesetzt sind. Um dies sicherzustellen, ist lediglich der unentgeltliche Einbau eines entsprechenden Messmoduls im Kühlschrank erforderlich. Dieser Sensor misst regelmäßig die Innentemperatur des Kühlschranks. Sobald diese innerhalb eines Zeitraums von 15 Minuten mehrfach vom vorgegebenen Normbereich abweicht, wird ein Signal weitergegeben und die Praxis umgehend per SMS und E-Mail informiert.

Nach Informationen von a1.direkt, Lorsch 